OBSERVATION OF CHARGE COMPENSATED POLARIZATION ZONES IN POLYVINYLIDENEFLUORIDE (PVDF) BY PIEZOELECTRICALLY GENERATED PRESSURE STEP WAVE (PPS) RESPONSE

$$
\text { W. Eisenmenger, M. Haardt, K. Holdik }
$$

Physikalisches Institut der Universität Stuttgart, D-7000 Stuttgart 80, Pfaffenwaldring 57, Germany

Experiment

Recently, measurements of the acoustic step-wave response using piezoelectric pressure or velocity-pulse excitation $[1],[2]$ have been performed with incompletely poled PVDFsamples.

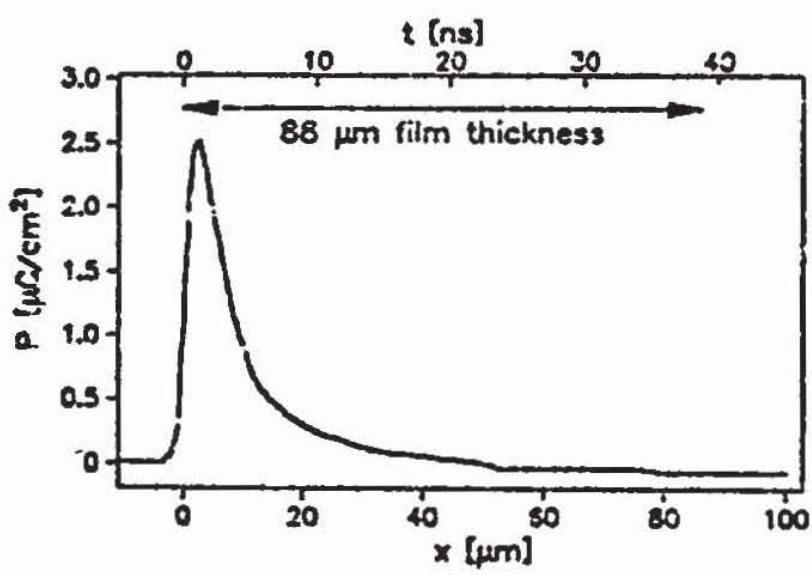

Fig. 1

Acoustic step response signal $I_{s}(t)$ of a thermalis poled $88 \mu \mathrm{m}$ thick PVDF film.

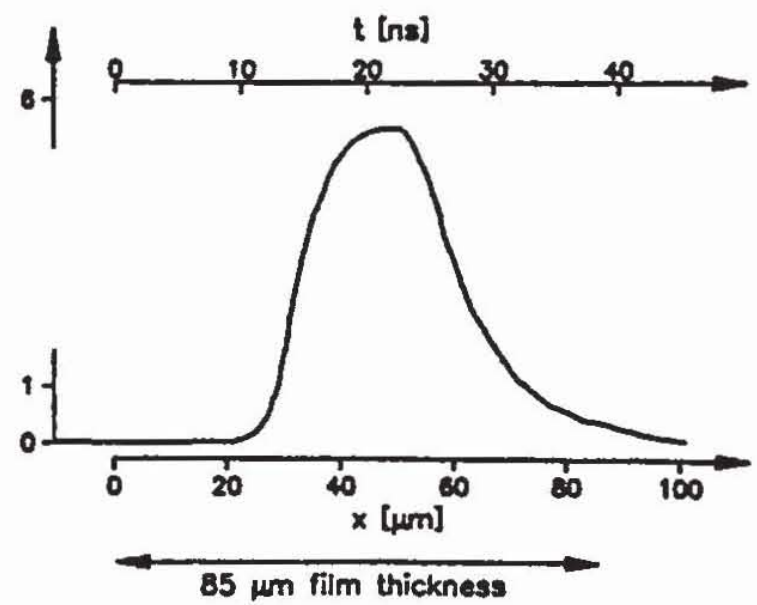

Fig. 2

Acoustic step response signal for a corona poled PVDF film. Polarization evaluated according to Ref. [1], [2].

We observed, see Fig. 1, a piezoelectric active layer at the positive poling electrode in agreement with the results of other authors [3], [4] for thermally poled films; 
whereas in films poled by corona discharge, see Fig. 2, an internal piezoelectric active zone without signal contributions at the film boundaries has been found. For samples with reduced conductance a significantly wider active zone, extending to the sample boundaries, see Fig. 3, under otherwise unchanged poling conditions, has been observed. In addition, higher conductance samples

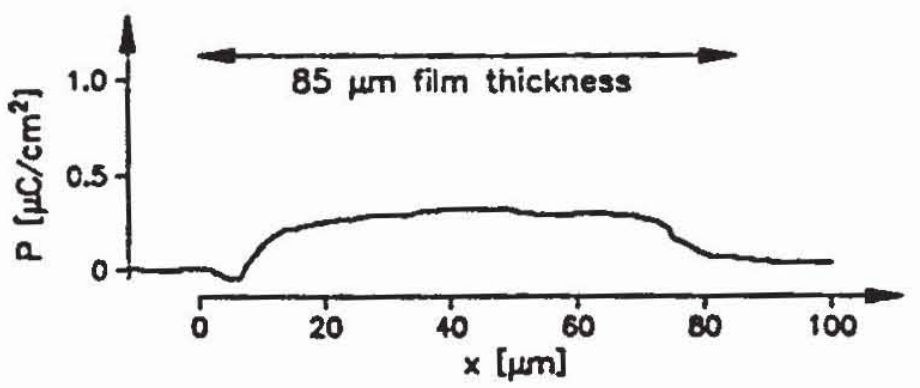

Fig. 3 Acoustic step response signal for a corona poled PVDF film with high insulation.

showed also [1] the development of double zones in the film interior. For long time high voltage poling with thin, appr. 20 to $50 \mu \mathrm{m}$ thick films, we observed almost homogeneous piezoelectric activity, as other authors did. The experimental result of Fig. 2 has been interpreted $[1],[2]$ as evidence for a space charge compensated persistent polarization zone. It is, of course, difficult to imagine why a sample should pole only in the center. In the following we discuss a theoretical model explaining this possibility.

Qualitative Model

Since PVDF is a poor insulting material with a dielectric-conductance relaxation time of the order of $100 \mathrm{sec}$ [5] charge transport in the poling procedure cannot be neglected. Corona discharge poling corresponds to constant current density conditions as long as the voltage developed across the dielectric film sample is smaller than the voltage between the corona point electrode and the ground. The initial development of charge transport in the film, therefore, is as follows: As consequence of finite conductance, \pm charges can be injected from both sides. Charges of opposite sign, approaching each other in the film center, increase the polarizing field. If polarization gives rise to charge-trapping, the conductance of the central zone is reduced by polarization, increasing the central field strength, the amount of charge-trapping, and the resistance of the central zone. This kind of feed-back mechanism then produces a high resistance-central zone being charged like an internal capacitor by the corona current. Since it is known that 
polarized PVDF-samples exhibit a reduced conductance [6], as compared to unpolarized material, our model appears quite natural.

\section{Quantitative Model}

In order to develop a quantitative model, describing polarization in the center of the sample, we introduce the following assumptions: 1 . Poling procedure and charge transport correspond to the so-called relaxation case [7], i.e. carrier lifetimes with respect to recombination are short compared to the dielectric-conductance relaxation time. From this follows that mainly excess carriers are important for charge-transport properties. Therefore, different regions can be treated separately by using only one carrier in each space regime. 2. the number of deep traps is directly proportional to the polarization. Micro scopically this can be attributed to charge trapping by dipole ends at the surfaces of crystallites in PVDF. With these assumptions current transport and polarization development are described by the Maxwell current continuity equation as follows:

$$
\text { jtot }=n_{f} q \mu E+\dot{D}
$$

with $j_{\text {tot }}=$ total injection current density; $n_{f}=$ density of free charge carriers; $q=$ charge of the carriers, $\mu=$ mobility; $E=$ electric field strength; $D=\varepsilon \varepsilon_{0} E+P(E)=$ total dielectric displacement density with $\varepsilon$ average reversible dielectric constant; $\varepsilon_{0}=$ vacuum permittivity; $P(E)=\varepsilon_{0} X_{P}=$ average crystallite polarization distribution with $X P=$ susceptibility assumed as constant for simplicity. In addition, dielectric continuity in our one-dimensional case corresponds to:

$$
\partial D / \partial x=q\left(n_{f}+n_{t}\right)
$$

Finally, the charge trapping process is described by:

$$
n_{t}=n_{f}\left(n_{0}-n_{t}\right) / n_{0} \tau_{t}
$$

with $n_{t}=$ density of trapped charges, $\dot{n}_{t}=$ time derivative of $n_{t} ; \tau_{t}=$ time constant of charge trapping;

$$
\mathrm{n}_{\mathrm{O}}=\mathrm{aP}
$$

$\mathrm{n}_{\mathrm{O}}=$ density of traps caused by polarization in linear approximation, as the most simple case.

The Stationary Solution

From Eqs. (1), (2) and (3) the stationary solution for the field strength $\mathrm{E}$ with $\dot{D}=0, \dot{x}_{t}=0$ and, therefore, 
$n_{0}=n_{t}$; can be obtrined by integration; c.f. Fig. 4:

$$
\left.E / E_{S}=P / P_{S}=y=\sqrt{b\left(e^{x} / \delta\right.}-1\right)
$$

with $\mathrm{P}_{\mathrm{S}}=$ saturation polarization;

$\mathrm{b}=\mathrm{j}_{\text {tot }} 2 \delta \varepsilon_{\mathrm{o}} \mathrm{X}_{\mathrm{P}}{ }^{2} / \mu \mathrm{P}_{\mathrm{S}}{ }^{2}\left(\varepsilon+\chi_{\mathrm{P}}\right)$ with $2 \delta=\left(\varepsilon+\chi_{\mathrm{P}}\right) / \mathrm{qa} \mathrm{XP}_{\mathrm{P}}$

a length approximately equal to the average crystallite dimension $d_{k}$. This relation corresponds to a quantitative determination of the proportionality constant " $\mathrm{a}$ " in Eq. (4) and is explained as follows: We assume that the crystallite polarization can be approximated by a certain number of completely oriented hypothetic "dipole chains" of the length $d$ parallel to $E$, at right angles to the molecular axis. Each orientated "dipole chain" carries one trap at its end with the dipole charge opposite to the free carrier charge. From this follows: $P=n_{0} q d_{k}$ or $a=1 / q d_{k}$, since the polarization contribution of each chain (or each trap) is equal to $\mathrm{qd}_{\mathrm{k}}$. The more correct expression of Eq. (6) describes the additional influence of the amorphous crystallite surrounding. Inserting typical data for $\mathrm{b}$ with $j$ tot $=1 \mu \mathrm{A} / \mathrm{cm}^{2} ; 2 \delta=10 \mu \mathrm{m}$; $\mu=10^{-6} \mathrm{~cm}^{2} /$ Vsec: $P_{S}=10 \mu \mathrm{C} / \mathrm{cm}^{2} ; \varepsilon=2$; $\mathrm{XP}_{\mathrm{P}}=10$; we obtain: $b=7.5 \cdot 10^{6}$. Comparing this number with the evaluation of Eq. (5) in Fig. 4; i.e. the stationary solution of the polarization in the dielectric film; we obtain internal polarization. A corresponding solution exists for the dominant (relaxation case) charge-carrier of opposite sign injected from the other side of the sample. If, of course, both solutions meet in the center, the situation is more complicated than represented by our simple model.

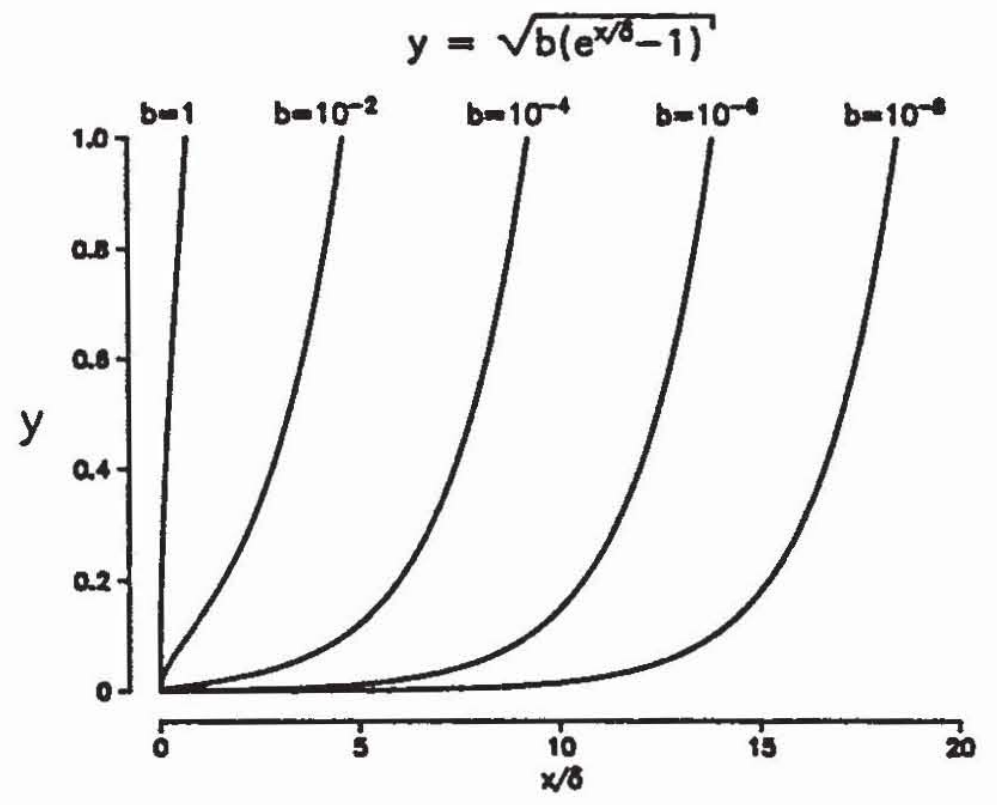

Fig. 4

Stationary field distribution according to $\mathrm{Eq}$. with internal polarization; $\mathrm{y}=\mathrm{E} / \mathrm{E}_{\mathrm{S}}=\mathrm{P} / \mathrm{P}_{\mathrm{S}}$ with $P_{S}=$ saturation polarization; $2 \delta \cong$ crystallite dimension. 
Fig. 4 also shows that for significantly higher values of the parameter $b$, the stationary solution changes from the internal to the more homogeneous polarization and a field profile with $E \propto \sqrt{x / \delta}$. This distribution is to be expected with high injection current and low mobility.

\section{Instationary Solution}

The complete time-dependent differential equation obtained from Eqs. $(1,2,3$ and 4$)$ with normalized field strength $\mathrm{y}=\mathrm{E} / \mathrm{E}_{\mathrm{S}}$ is:

$2 \delta \tau_{t} y^{3} \dot{y}^{\prime}+\tau_{g} \tau_{t} y^{2} \ddot{y}=\left(b-\dot{y} \tau_{g}\right)\left(-\tau_{t} y \dot{y}+y^{2}-2 \delta y y^{\prime}+b-\dot{y} \tau_{g}\right)$

with: $\dot{y}=\partial y / \partial t ; y^{\prime}=\partial y / \partial x$; etc.; $\tau_{t}=$ time constant for trapping, c.f. Eq. (3); $\tau_{g}=E_{s} b \varepsilon_{o}\left(\varepsilon+x_{p}\right) / j_{\text {tot }}=$ time constant for polarization growth. All other constants see Eqs. (5 and 6). For the stationary case, the second right hand bracket results in $y^{2}-2 \delta y y^{\prime}+b=0$ with the solution given in Eq. (5). Experimentally we observed [1] as nonstationary situation that the central polarization-zone amplitude grows linearly with time. This behavior is qualitatively described by Eq. (7). Assuming a nonstationary solution with an almost rectangular shape of the internal polarization zone, growing linearly in time, the left hand side of Eq. (7) vanishes with the exception of an edge singularity. On the right side a linear growth is described if the first bracket equals zero, resulting in $\dot{y}=j_{\text {tot }} / E_{S} \varepsilon_{0}\left(\varepsilon+\chi_{P}\right)$; i.e. quantitatively in agreement with the pure dielectric charging process. Outside the growing zone the polarization is small and shows little change with time, therefore, the second bracket must vanish. This corresponds to the stationary solution. It is important to note that, for the nonstationary solution, the density of trapped charges is not necessarily proportional to the polarization, since traps can remain empty.

\section{Discussion}

Comparing experiment with the calculation, the 30 minutes thermally poled sample in Fig. 1 shows good qualitative agreement with the calculated data of Fig. 4. We expect that the stationary solution has been reached. Within our model, the experimental result can be explained by the transport of negative charge-carriers from the negative to the positive electrode.with a mobility of at least $10^{-6} \mathrm{~cm}^{2} /$ Vsec. Positive charges have either an extremely low mobility or in case of high mobility cannot be in- 
jected from the positive electrode. The experimental result of Fig. 3 with a wide polarization distribution, indicating $P \propto \sqrt{x}$ at the film boundaries, corresponds to the curve with high b-value in Fig. 4 or to the condition of high current injection and low mobility. In agreement with this we found a higher specific resistance for the sample of Fig. 3 than for the sample of Fig. 2. In the case of central polarization zones, c.f. Fig. 2, we observed additional instable behavior [1] in which a central poling zone, after reaching maximum polarization, decays to zero followed by the development of a double polarization zone. We expect that this behavior is related to the often observed instabilities during the first poling cycle in hysteresis experiments [8], and can be studied by acoustic step-wave measurements during the poling process [2].

Financial support by the Deutsche Forschungsgemeinschaft is gratefully acknowledged.

\section{References}

1. W.Eisenmenger, M.Haardt: Sol. State Com. 41, 917, (1982)

2. M.Haardt, W.Eisenmenger, this Volume

3. R.J.Phelan et al., Ferroelectrics I, 375, (1974)

4. H.Sussner, K.Dransfeld: J. Polymer Science. Polymer Physics, Ed. Vol. 16, 529-543,(1978)

5. For a general review see "Electrets" (Edited by G.M. Sessler), Topics in Applied Physics, Vol. 33, Springer Verlag, Berlin (1980)

6. G.Pfister et al., J. Appl. Phys. 4 4, 2064, (1973)

7. H.J. Queisser: Solid State Devices, 1972, Ed. by P.N.Robson, Conf. Ser. 15, Inst. of Phys., LondonBristol, P. 145, (1973)

8. See for example J.E.McKinney et al. J. Appl. Phys. 51, 1676, (1980) 